
\title{
$\begin{array}{ll}\text { Research Square } & \begin{array}{l}\text { Preprints are preliminary reports that have not undergone peer review. } \\ \text { They should not be considered conclusive, used to inform clinical practice, } \\ \text { or referenced by the media as validated information. }\end{array}\end{array}$
}

\section{The Genetic Structure of Pain in Depression Patients: A Genome-Wide Association Study and Proteome-Wide Association Study}

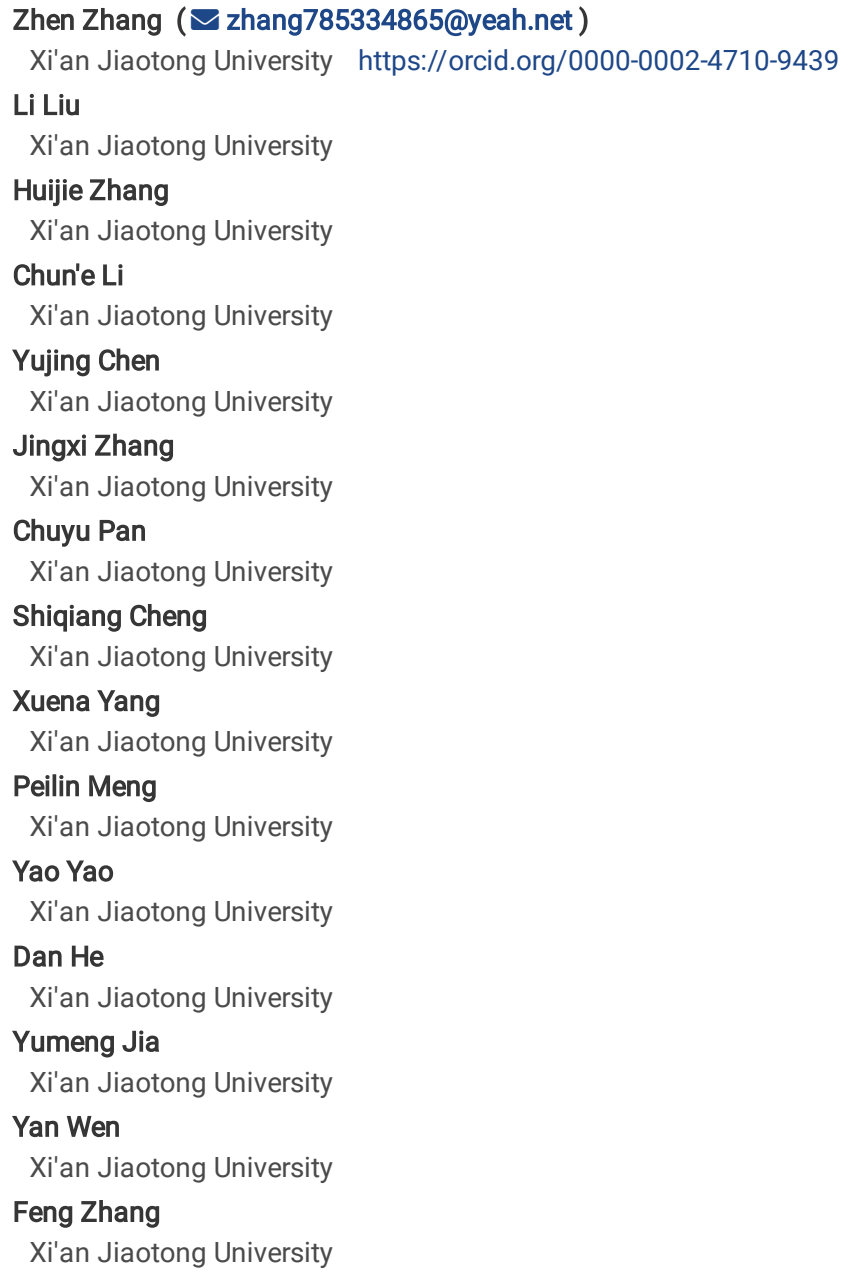

\section{Research article}

Keywords: depression, pain, multisite chronic pain, genome-wide association study, linkage disequilibrium score regression analysis, proteome-wide association study.

Posted Date: January 4th, 2022

DOI: https://doi.org/10.21203/rs.3.rs-1210160/v1

License: (c) (i) This work is licensed under a Creative Commons Attribution 4.0 International License. Read Full License 


\section{Abstract \\ Background}

Pain symptoms are common in the patients with depression. Comparing with the general population, the pain in depression patients has more complex biological mechanism. We aim to explore the etiological mechanism of pain in depression patients from the perspective of genetics.

\section{Methods}

Utilizing the UK Biobank samples with self-reported depression status or PHQ score $\geq 10$, we conducted genome-wide association study (GWAS) of seven pain traits $(N=1,133-58,349)$. The GWAS summary were then integrated with two different reference protein weights (ROS/MAP and Banner) for proteome-wide association study (PWAS) using the FUSION pipeline. Additionally, LDSC analysis was performed to explore the genetic correlation between pain traits in depression patients and common psychiatry disorders. And biological processes and functions that related to pain associated genes in depression patients were analyzed by gene set enrichment analysis.

\section{Results}

GWAS identified 3 significant genes associated with different pain traits in depression patients, including $T R I O B P\left(P_{\text {GWAS }} 4.48 \times 10^{-8}\right)$ for stomach or abdominal pain, SLC9A9 $\left(P_{\text {GWAS }}=2.77 \times 10^{-8}\right)$ for multisite chronic pain (MCP) and ADGRF1 $\left(P_{\text {GWAS }} 1.51 \times 10^{-8}\right)$ for neck or shoulder pain. PWAS also identified multiple candidate genes associated with different pain traits in depression patients, such as TPRG1L (permutation-based $P_{\mathrm{PWAS}-\mathrm{Banner}}=3.38 \times$ $10^{-2}$ ) and SIRPA (permutation-based $P_{\mathrm{PWAS}-\text { Banner }}=3.65 \times 10^{-2}$ ) for MCP etc. LDSC analysis results showed that MCP was positively correlated with attentiondeficit hyperactivity disorder (ADHD) (genetic correlation $\left.(r g)=0.123, P_{\text {LDSC }}=0.039\right)$ and post-traumatic stress disorder $($ PTSD $)\left(r g=0.217, P_{\text {LDSC }}=0.029\right)$.

\section{Conclusions}

We reported multiple novel candidate genes and genetic correlations for pain traits in depression patients, providing novel clues for understanding the genetic mechanisms underlying the pain in depression patients.

\section{Introduction}

As one of the most common psychiatric disorders, depression not only causes changes in emotional state (affective disorder), but also usually causes major physical symptoms such as primarily fatigue, pain or sleep disturbances. ${ }^{1}$ According to the World Health Organization's latest report, the prevalence of depression is steadily increasing worldwide, with an estimated 322 million people (4.4\% of the world's population) living with depression. ${ }^{2}$ Furthermore, depression is the single largest contributor to nonfatal health loss globally, accounting for $7.5 \%$ of all years of disability in $2015 .{ }^{2}$ As a complex disease, depression not only reduces the health-related quality of life of patients, and increase the economic burden of individuals and social medical economic burden, which has become a public health problem of global concern. ${ }^{3,4}$

Pain is an unpleasant sensory and emotional experience associated with actual or potential tissue damage, and is an interaction of psychological, emotional, behavioral and social factors. ${ }^{5}$ Although pain is not a diagnostic symptom of depression, pain symptoms are common in people with depression and are closely related to depression. Previous studies have found that the prevalence of pain in depression patients was $59.1 \%(95 \% \mathrm{Cl}: 57.7 \%-60.7 \%)^{6}$, and pain occurred more frequently in patients with depression than in those without. ${ }^{7}$ A longitudinal study that excluded participants with a history of depression or anxiety showed that baseline pain symptoms were a major risk factor for depression. ${ }^{8}$ What's more, previous studies have shown that pain can adversely affect the prognosis and treatment of depression. For example, a systematic review indicates that patients with both pain and depression had reduced physical, mental and social functioning as opposed to those with depression or pain alone. ${ }^{9}$

Previous research on between depression and pain symptoms has shown that because of the complex interaction between depression and pain symptoms, multiple common neurobiological mechanisms, genetics, environmental and cognitive factors must be considered when trying to unravel their association. ${ }^{10}$ One widely accepted theory holds that depression and painful symptoms follow the same descending pathways of the central nervous system. That is, in the nerves of the downward pain regulation system, key structures of the brainstem pain regulation circuit (periaqueductal gray(PAG) and rostralventromedial medulla(RVM)) receives many projections from the brain regions involved in the production of emotions (medial prefrontal cortex, insular cortex(IC), prefrontal cortex(PFC), hypothalamus and amygdala), and jointly constructs the pathway of pain regulation. ${ }^{11}$ In addition, previous studies have found that depression and pain, specifically chronic pain, share common pathophysiological pathways that contribute to the long-term existence of depression, such as inflammatory processes, hypothalamic-pituitary-adrenocortical axis abnormalities and altered neurotransmitter receptivity in descending pathways of the central nervous system. ${ }^{10,12}$ These common neural circuits and neurochemicals enable depression and pain symptoms to interact at multiple levels within and between brain regions, between cells, and through neurochemical signals, where changes in circuit activity and neurochemical levels caused by either side can affect the other. Due to the complex interaction of these two diseases, compared with the general population's pain symptoms, the pain in depression patients has more complex biological mechanism, and depression itself is more serious, longer course of disease, and worse prognosis. Thus, it is necessary to pay attention to the pain in patients with depression and propose more effective strategies for the treatment and prognosis of depression. 
It is worth noting that several previous studies have emphasized individual differences in susceptibility to depression and pain responses, indicating that genetic factors may have some common roles in the pathogenesis of depression and pain. ${ }^{12,13}$ Multivariate models of a twin study estimated $39 \%$ (95\% Cl: $22 \%-53 \%)$ heritability for depression and $25 \%$ (95\% Cl: $9 \%-41 \%$ ) heritability for pain. ${ }^{14}$ Meng et al. conducted the cross-trait linkage disequilibrium score regression (LD) analysis for different pain phenotypes, and identified significant and positive genetic associations of multiple pain phenotypes with depression and neuroticism, revealing that pain and depression have partially overlapping genetic risk factors. ${ }^{15}$ Moreover, a genome-wide association study (GWAS) of multisite chronic pain based on data from the UK Biobank (UKB) cohort identified 39 susceptibility genes significantly associated with chronic pain, including DCC, MAML3 and FOXP2, with functions implicated neuronal development, neurogenesis and neural connectivity. ${ }^{16}$ Summary of previous studies on depression and pain symptoms have found that due to the complex interactions and common genetic risk factors between them, there may be differences in the biological mechanism of pain symptoms between depression patients and the general population. However, few studies have conducted GWAS of pain in depression patients, and the corresponding susceptibility genes are unknown.

Utilizing the UK Biobank cohort, we conducted GWAS of different pain traits in depression patients. Then, the GWAS summary data were further subjected to PWAS and LDSC analysis in order to explore the potential biological mechanism related to pain in depression patients from the perspective of genetics. Finally, gene enrichment analysis was conducted to further reveal the possible biological pathway of genes related to pain phenotype in patients with depression. It is hoped to provide a new direction for the etiological mechanism study of depression and pain symptoms.

\section{Materials And Methods}

\section{UK Biobank Cohort}

The UK Biobank is a large prospective cohort study that collected health-related information, including phenotypes and genotypes, from approximately 500,000 participants aged between 40 and 69 years from around the United Kingdom in 2006 and $2010 .{ }^{17}$ All participants were asked to report a range of demographic information and health status through questionnaires and interviews, and approved to use their anonymous data for any health-related research. The UK Biobank provides informed consent to all participants. Our study was approved by the UK Biobank (Application 46478) and health-related records of participants were obtained. ${ }^{17}$

\section{Depression Patients from UK Biobank}

According to the definition of depression phenotype in the previous study, ${ }^{18}$ we selected the depression patients from the participants of UK Biobank cohort. Briefly, the depression patients were determined based on self-report depression status (UK Biobank data fields: 20002, 20126, and 20544) or Patient Health Question-9 (PHQ) score $\geq 10$. For self-reported depression, depression patients were selected based on the code 1286 from ID 20002 , code 3,4 or 5 from ID 20126 and code 11 from ID 20544. In order to classify depression patients as closely as possible, we used the Patient Health Question-9 (PHQ) ${ }^{19}$ as strict inclusion and exclusion criteria. PHQ is a classification scale with an overall score (0-27) that focuses on nine symptoms and signs of depression and is used to screen and measure the severity of depression. Detailed classification of depression is presented in the Supplementary Information. Our analysis was limited to white British individuals (UK biobank data domain :21000).

\section{Pain Phenotypes of UK Biobank}

According to the specific pain related questionnaire adapted by the UK Biobank, the pain phenotype was measured by a question: "whether the participant reported having suffered from various types of body pain in the previous 3 months? The options were: 1 . headache, 2. facial pain, 3 . neck or shoulder pain, 4. back pain, 5. stomach or abdominal pain, 6. hip pain, 7. knee pain, 8. pain all over the body, 9. none of the above. More than one option could be selected (UK Biobank Category ID 100048)." Eight different pain phenotypes were defined based on pain at different sites reported by participants for the past 3 months or more, and complete definitions of the eight pain phenotypes can be found in the supplementary information. For each pain phenotype, cases were defined as people who chose the option of having pain at a specific site for 3 months or more in response to the above question. The controls were those who chose "none of the above" or "pain in a specific site did not last 3 months." Due to the small sample size for general pain for 3+ months (Field ID:2956) and facial pains for 3+ months (Field ID:4067), our study excluded these two pain phenotypes. In addition, we defined multisite chronic pain (MCP) based on the UK Biobank pain phenotype of seven different body sites with a pain score questionnaire (score 0-7). Once participants chose the most recent pain in one or more body sites or all over the body, they were also asked if the pain had been ongoing for three months or more (defined as chronic pain). Thus, MCP is defined as the total number of body sites that record chronic pain (lasting at least 3 months) :0 to 7 . Those who chose "pain over the body" were excluded from our study because of the difference between widespread pain and localized chronic pain. ${ }^{16}$

\section{Genotyping, Imputation and Quality Control of UK Biobank}

In UK Biobank cohort, a total of 488,377 participants were genotyped by either the Affymetrix UK BiLEVE Axiom array or the UK Biobank Axiom array. ${ }^{20}$ Subsequently, these genotypes results were imputed and quality controlled based on the Haplotype Reference Consortium (HRC) reference panel and UK10K project Reference panels. ${ }^{21}$ The details of array design, genotyping and quality control procedures are described elsewhere. ${ }^{22}$ Then, we deleted those participants who reported discrepancies between genetic gender and self-reported gender, as well as without ethical consent and interpolation data.

Additionally, we excluded variants with the Hardy-Weinberg equilibrium (HWE) test $P$-values $>1.0 \times 10^{-5}$, a genotype missing rate of $>0.05$ and a minor allele frequency (MAF) of $<0.01$. Ultimately, we used KING software (http://people.virginia.edu/ wc $9 \mathrm{c} / \mathrm{KING} /$ index.html) to exclude the genetically related individuals.

Genome-Wide Association Study (GWAS) 
We performed GWAS studies of seven different pain phenotypes in depression patients from the UK Biobank cohort. That is, the additive genetic effect logistic regression model of PLINK2.0 was used to conduct association analysis with adjusted sex, age, smoke consumption, alcohol consumption, body mass index(BMI), PC1, PC2 and PC3 as covariables. ${ }^{23}$ For quality control, we select high quality SNPs through a quality control filters: SNPs with low call rates $(<0.90)$, high SNP-level missingness $(>0.1)$, high individual-level missingness $(>0.1)$, low $\operatorname{MAF}(<0.01)$ and low HWE exact test $P$-values $(<0.001)$ were excluded. $P_{\text {GWAS }}<5.0 \times 10^{-8}$ and $P_{\text {GWAS }}<1.0 \times 10^{-5}$ were defined as significant and suggestive associations, respectively. GWAS results were visualized with the Manhattan plots generated by the "CMplot" R script. (https://github.com/YinLiLin/R-CMplot)

\section{Linkage Disequilibrium Score Regression (LDSC) Analysis}

According to the LDSC software(v1.0.0,https://github.com/bulik/ldsc), we used the cross-trait LDSC method to calculate the genetic correlation between different pain phenotypes and seven mental disorders ${ }^{24}$, including Alzheimer's disease $(A D)^{25}$, anxiety ${ }^{26}$, attention-deficit hyperactivity disorder(ADHD) ${ }^{27}$, autism spectrum disorder(ASD) ${ }^{28}$, bipolar disorder(BD ${ }^{29}$, major depressive disorder(MDD) ${ }^{30}$ and post-traumatic stress disorder(PTSD) ${ }^{31}$. The GWAS data of these seven psychiatric disorders were obtained from the published articles by the Psychiatric Genomics Consortium (PGC)

(https://www.med.unc.edu/pgc/download-results/). The LDSC analysis is not affected by sample overlap and is fast, making it a reliable and powerful method to calculate genetic correlations between different phenotypes using GWAS summary-level results data. ${ }^{24,32}$

\section{Proteome-Wide Association Study (PWAS)}

We separately integrated two different reference human brain proteomes(the ROS/MAP brain proteome reference weights ${ }^{33}$ and Banner brain proteome reference weights ${ }^{34}$ ) with our GWAS summary of pain in depression patients for PWAS using FUSION pipeline, respectively.Specifically, FUSION pipeline was used to calculate PWAS association statistics (PWAS Z-score), and then combined with the pre-calculated brain proteome reference weight (Z-score $\times$ proteome weight) to evaluate the effects of significant SNP in the pain GWAS of depression patients on the protein abundance. Finally, FUSION identifies the candidate genes associated with pain in patients with depression that act by regulating the abundance of proteins in the brain. To control the potential effect of multiple testing on study results, the permutation-based $P$-value (named PERM.PV) was calculated for each gene by FUSION. The permutation-based $P$ value $\left(P_{\text {PWAS }}\right)<0.05$ was used as significance threshold in our PWAS analysis.

Two different reference human brain proteomics used in our study were from Religious Orders Study and Rush Memory ${ }^{33}$ and Aging Project (ROS/MAP) and the Banner Sun Health Research Institute (Banner) ${ }^{34}$. According to previous study of human brain proteome ${ }^{35}$, proteome was sequenced and combined with significant SNPS in GWAS database to identify cis-regulated proteins that were significantly related to genetic variation. Detailed information on proteome sequencing, quality control, and standardization can be obtained in the study by Wingo et al. ${ }^{35}$ After analysis and quality control, the ROS/MAP proteome $(\mathrm{N}=376)$ identified a total of 1,475 proteins that showed a significant cis-association with genetic variation. The Banner proteome $(\mathrm{N}=152)$ identified 1,139 proteins that showed a significant cis-association with genetic variation.

\section{Functional exploration of identified candidate genes}

We used Metascape's online analytical tool (http://metascape.org) to explore the functions of genes identified by our GWAS and PWAS, including Gene Ontology (GO), pathway analysis. GO was used for gene function classification and annotation, Pathway Analysis was used to analyze a specific classical metabolic or regulatory network of genes, proteins or molecules. All genes in the genome are used as the enrichment background. The terms with $P$ value $<0.05$, minimum count of 3 , and enrichment factor $>1.5$ were collected, then group them into clusters based on the similarity of their members.

\section{Results}

\section{General population characteristics}

In our study, we analyzed seven different pain phenotypes in two depression samples, defined by self-reported depression status and PHQ score $\geq 10$. Table 1 details the general characteristics of study samples.

Table 1. General population characteristics of depression patients 


\begin{tabular}{|c|c|c|c|c|}
\hline Study samples & Pain phenotypes & $\begin{array}{l}\text { Number/ } \\
\text { (Case/Control) }\end{array}$ & Sex (Male) & Age $\pm S D$ \\
\hline \multirow{7}{*}{$\begin{array}{l}\text { Self-reported } \\
\text { depression }\end{array}$} & Headache & $8310 / 7742$ & $4765(29.68 \%)$ & $53.84 \pm 7.59$ \\
\hline & Neck or shoulder pain & $12062 / 4432$ & $5524(33.49 \%)$ & $55.56 \pm 7.64$ \\
\hline & Stomach or abdominal pain & $4287 / 2828$ & $2318(32.58 \%)$ & $53.94 \pm 7.97$ \\
\hline & Back pain & $13248 / 4636$ & $6418(35.89 \%)$ & $55.63 \pm 7.80$ \\
\hline & Hip pain & $6713 / 1622$ & $2378(28.53 \%)$ & $56.95 \pm 7.50$ \\
\hline & Knee pain & $11514 / 2669$ & $5091(35.90 \%)$ & $56.51 \pm 7.50$ \\
\hline & Multisite chronic pain & 58349 & $20345(34.87 \%)$ & $55.72 \pm 7.74$ \\
\hline \multirow[t]{7}{*}{ PHQ score $\geq 10$} & Headache & $1296 / 1066$ & $762(32.26 \%)$ & $51.90 \pm 7.31$ \\
\hline & Neck or shoulder pain & $1724 / 537$ & $839(37.11 \%)$ & $53.32 \pm 7.55$ \\
\hline & Stomach or abdominal pain & $744 / 390$ & $379(33.42 \%)$ & $51.90 \pm 7.64$ \\
\hline & Back pain & $1848 / 579$ & $951(39.18 \%)$ & $53.09 \pm 7.83$ \\
\hline & Hip pain & $944 / 189$ & $359(31.69 \%)$ & $54.65 \pm 7.62$ \\
\hline & Knee pain & $1556 / 301$ & $742(39.96 \%)$ & $53.98 \pm 7.54$ \\
\hline & Multisite chronic pain & 6593 & $2460(37.31 \%)$ & $53.13 \pm 7.67$ \\
\hline
\end{tabular}

Abbreviations: SD, age was described as mean \pm standard deviation (SD), PHQ score, patient health questionnaire (PHQ) is used to describe the depression.

\section{GWAS analysis results}

For self-reported depression samples, significant associations $\left(P_{\mathrm{GWAS}}<5.0 \times 10^{-8}\right)$ were found at the rs $12627973\left(P_{\mathrm{GWAS}}=4.48 \times 10^{-8}\right)$, rs $2627943\left(P_{\mathrm{GWAS}}=\right.$ $\left.4.73 \times 10^{-8}\right)$ and rs62236743 $\left(P_{\mathrm{GWAS}}=4.76 \times 10^{-8}\right)$ of $T R I O B P$ gene for the stomach or abdominal pain, as well as rs56154708 $\left(P_{\mathrm{GWAS}}=2.77 \times 10^{-8}\right)$ of SLC9A9 gene for MCP. Additionally, four SNPS of SLC9A9 gene showed suggestive association signals for MCP, including rs $10513209\left(P_{\mathrm{GWAS}}=9.19 \times 10^{-8}\right)$, rs765265245 $\left(P_{\text {GWAS }}=9.16 \times 10^{-8}\right), \operatorname{rs} 62269778\left(P_{\text {GWAS }}=9.07 \times 10^{-8}\right)$, and rs62269777 $\left(P_{\text {GWAS }}=9.06 \times 10^{-8}\right)$.

For the depression patients with $\mathrm{PHQ}$ score $\geq 10$, significant associations were detected at rs $201595898\left(P_{\mathrm{GWAS}}=1.51 \times 10^{-8}\right)$, rs $45511696\left(P_{\mathrm{GWAS}}=1.63 \times\right.$ $\left.10^{-8}\right)$ and rs $45598235\left(P_{\text {GWAS }}=1.64 \times 10^{-8}\right)$ of $A D G R F 1$ gene for the neck or shoulder pain. The visualization of the results is shown in Figure 1 . Other detailed results are shown in Table 2.

Table 2. GWAS results of different pain phenotypes in depression patients.

\begin{tabular}{|c|c|c|c|c|c|c|c|}
\hline Objects & Pain phenotypes & SNP & CHR & Gene & OR & SE & $P$-Value \\
\hline \multirow{9}{*}{$\begin{array}{l}\text { Self-reported } \\
\text { depression }\end{array}$} & \multirow[t]{5}{*}{ Multisite chronic pain } & rs56154708 & 3 & SLC9A9 & 0.932 & 0.013 & $2.77 \times 10^{-8}$ \\
\hline & & rs10513209 & 3 & SLC9A9 & 0.935 & 0.013 & $9.19 \times 10^{-8}$ \\
\hline & & rs765265245 & 3 & SLC9A9 & 0.933 & 0.013 & $9.16 \times 10^{-8}$ \\
\hline & & rs62269778 & 3 & SLC9A9 & 0.935 & 0.013 & $9.07 \times 10^{-8}$ \\
\hline & & rs62269777 & 3 & SLC9A9 & 0.935 & 0.013 & $9.06 \times 10^{-8}$ \\
\hline & \multirow[t]{4}{*}{ Stomach or abdominal pain } & rs12627973 & 22 & TRIOBP & 0.745 & 0.054 & $4.48 \times 10^{-8}$ \\
\hline & & rs1004890 & 22 & - & 0.745 & 0.054 & $4.57 \times 10^{-8}$ \\
\hline & & rs12627943 & 22 & TRIOBP & 0.746 & 0.054 & $4.73 \times 10^{-8}$ \\
\hline & & rs62236743 & 22 & TRIOBP & 0.746 & 0.054 & $4.76 \times 10^{-8}$ \\
\hline \multirow[t]{3}{*}{ PHQ score $\geq 10$} & \multirow[t]{3}{*}{ Neck or shoulder pain } & rs201595898 & 6 & ADGRF1 & 0.305 & 0.209 & $1.51 \times 10^{-8}$ \\
\hline & & rs45511696 & 6 & ADGRF1 & 0.318 & 0.203 & $1.63 \times 10^{-8}$ \\
\hline & & rs45598235 & 6 & ADGRF1 & 0.318 & 0.203 & $1.64 \times 10^{-8}$ \\
\hline
\end{tabular}

Abbreviations: SNP, single nucleotide polymorphism, CHR, chromosome, OR, odds ratios, $\mathrm{SE}$, standard error, $\mathrm{PHQ}$ score, patient health questionnaire ( $\mathrm{PHQ}$ ) is used to describe the depression. $P_{\mathrm{GWAS}}<5.0 \times 10^{-8}$ and $P_{\mathrm{GWAS}}<1.0 \times 10^{-5}$ were defined as significant and suggestive associations, respectively. 


\section{LDSC analysis results}

LDSC analysis identified multiple genetic correlations between pain traits and mental disorders, such as MCP and ADHD (genetic correlation $(\mathrm{rg})=0.123, P_{\mathrm{LDSC}}$ $=0.039), \mathrm{MCP}$ and PTSD $\left(\mathrm{rg}=0.217, P_{\mathrm{LDSC}}=0.029\right)$ in self-reported depression patients. In addition, neck or shoulder pain $\left(\mathrm{rg}=0.222, P_{\mathrm{LDSC}}=0.001\right)$ and MCP $\left(r g=0.223, P_{\text {LDSC }}=0.012\right)$ were correlated with ADHD in depression patients with PHQ score $\geq 10$. Table 3 showed the genetic associations between different pain phenotypes and seven psychiatric disorders in depression populations.

Table 3. The genetic correlation between different pain phenotypes and seven psychiatric disorders in depression patients.

\begin{tabular}{|c|c|c|c|c|c|c|c|c|c|c|c|c|c|c|c|}
\hline \multirow[t]{2}{*}{ Objects } & \multirow{2}{*}{$\begin{array}{l}\text { Pain } \\
\text { phenotypes }\end{array}$} & \multicolumn{2}{|l|}{$A D$} & \multicolumn{2}{|c|}{ Anxiety } & \multicolumn{2}{|l|}{ ADHD } & \multicolumn{2}{|l|}{ ASD } & \multicolumn{2}{|l|}{ BD } & \multicolumn{2}{|l|}{ MDD } & \multicolumn{2}{|l|}{ PTSD } \\
\hline & & $\mathrm{rg}$ & $P$ & $\mathrm{rg}$ & $P$ & $\mathrm{rg}$ & $P$ & $\mathrm{rg}$ & $P$ & $\mathrm{rg}$ & $P$ & $\mathrm{rg}$ & $P$ & $\mathrm{rg}$ & $P$ \\
\hline \multirow{2}{*}{$\begin{array}{l}\text { Self- } \\
\text { reported } \\
\text { depression }\end{array}$} & Knee pain & 0.309 & 0.256 & 0.388 & 0.375 & 0.109 & 0.572 & 0.475 & 0.302 & 0.029 & 0.821 & 0.039 & 0.685 & 0.0061 & $0.97 \varepsilon$ \\
\hline & $\begin{array}{l}\text { Multisite } \\
\text { chronic } \\
\text { pain }\end{array}$ & 0.111 & 0.222 & 0.191 & 0.201 & 0.123 & 0.039 & 0.053 & 0.361 & 0.019 & 0.683 & 0.083 & 0.050 & 0.217 & 0.02 \\
\hline \multirow[t]{3}{*}{$\begin{array}{l}\text { PHQ score } \\
\geq 10\end{array}$} & $\begin{array}{l}\text { Neck or } \\
\text { shoulder } \\
\text { pain }\end{array}$ & 0.135 & 0.243 & 0.263 & 0.221 & 0.222 & 0.001 & 0.100 & 0.124 & 0.069 & 0.204 & 0.026 & 0.564 & 0.002 & 0.98 \\
\hline & $\begin{array}{l}\text { Stomach or } \\
\text { abdominal } \\
\text { pain }\end{array}$ & -0.083 & 0.597 & -0.244 & 0.651 & 0.035 & 0.842 & -0.067 & 0.790 & -0.111 & 0.488 & 0.059 & 0.433 & -0.093 & $0.64 c$ \\
\hline & $\begin{array}{l}\text { Multisite } \\
\text { chronic } \\
\text { pain }\end{array}$ & 0.661 & 0.048 & 0.120 & 0.285 & 0.223 & 0.012 & 0.077 & 0.367 & 0.027 & 0.676 & 0.105 & 0.056 & -0.078 & $0.50 \varepsilon$ \\
\hline
\end{tabular}

Abbreviations: AD, alzheimer's disease, ADHD, attention-deficit hyperactivity disorder, ASD, autism spectrum disorder, BD, bipolar disorder, MDD, major depressive disorder, PTSD, post-traumatic stress disorder, SCZ, schizophrenia. PHQ score, patient health questionnaire (PHQ) is used to describe the depression. rg, genetic correlation. $\mathrm{P}$ values $<0.005$ were considered as significant for genetic correlations. Those significant $\mathrm{P}$ values were in bold.

\section{PWAS analysis results}

Figure 2 and 3 summarizes the results of PWAS of different pain traits in depression patients. For PWAS with Banner reference weights, a total of 15 genes related to cis-regulated brain protein abundance were identified for the stomach or abdominal pain, such as $M L C 1\left(P_{\mathrm{PWAS}-\mathrm{Banner}}=4.80 \times 10^{-3}\right)$ and $\operatorname{GSTM} 3\left(P_{\mathrm{PWAS}-\mathrm{Banner}}=3.38 \times 10^{-2}\right)$, etc. And 17 significant related genes were identified for MCP, such as $\operatorname{SIRPA}\left(P_{\mathrm{PWAS}-\mathrm{Banner}}=3.65 \times 10^{-2}\right)$ and TPRG1L $\left(P_{\mathrm{PWAS}-\mathrm{Banner}}=3.38 \times 10^{-2}\right) .8$ genes were identified for the neck or shoulder pain, such as $M C E E\left(P_{\mathrm{PWAS}-\mathrm{Banner}}=6.00 \times 10^{-4}\right)$ and $P 2 R X 7\left(P_{\mathrm{PWAS}-\mathrm{Banner}}\right.$ $=3.54 \times 10^{-2}$ ). Comparing the significant genes, there were 2 overlapping genes (GSTM3 and MLC1) identified for both the stomach or abdominal pain phenotype and MCP, while 1 overlapping gene (MCEE) for stomach or abdominal pain and neck or shoulder pain, as well as 1 overlapped gene (NAV1) for MCP and neck or shoulder pain.

For PWAS using Rosmap reference weights, multiple genes were also identified, such as MICAL $1\left(P_{\mathrm{PWAS} \text {-Rosmap }}=3.43 \times 10^{-3}\right)$ and $S T A M 2\left(P_{\mathrm{PWAS}-\text { Rosmap }}=\right.$ $\left.1.19 \times 10^{-2}\right)$ for stomach or abdominal pain phenotype, SIRPA $\left(P_{\mathrm{PWAS}-\text { Rosmap }}=2.24 \times 10^{-2}\right)$ and TPRG1L $\left(P_{\mathrm{PWAS} \text {-Rosmap }}=3.29 \times 10^{-2}\right)$ for MCP, $S L C 30 A 9\left(P_{\mathrm{PWAS}}\right.$ Rosmap $\left.=2.78 \times 10^{-3}\right)$ and MRPL $10\left(P_{\mathrm{PWAS} \text {-Rosmap }}=4.30 \times 10^{-2}\right)$ for neck or shoulder pain phenotype .Comparing the significant genes found 1 overlapping gene (ARHGEF10) identified for both stomach or abdominal pain and MCP.

We also compared above PWAS results using respectively the Banner and Rosmap reference weights. We found two overlapping genes, SIRPA ( $P_{\mathrm{PWAS}}{ }^{\circledR} \mathrm{Banner}=$ $3.65 \times 10^{-2}, P_{\text {PWAS }}$ Rosmap $\left.=2.24 \times 10^{-2}\right)$ and TPRG1L $\left(P_{\text {PWAS Banner }}=3.38 \times 10^{-2}, P_{\text {PWAS Rosmap }}=3.29 \times 10^{-2}\right)$ for MCP. Detailed PWAS analysis results can be found in Supplementary Information Table 3.

\section{Functional exploration results}

After pathways and processes enrichment analysis of the significant genes identified by GWAS and PWAS, a total of 56 GO terms were obtained. These GO terms are mainly involved in sulfur compound metabolic process, regulation of actin filament organization, tRNA metabolic process, signal release, chemical synaptic transmission et al. The biological processes or functional pathways of the 16 clusters detected in the enrichment analysis were summarized by the Bubble plot, and the detailed results were shown in Figure 4.

\section{Discussions}

Previous studies on pain symptoms have suggested that pain mainly involves peripheral, spinal and central effects, including various factors that affect nociceptive perception, inflammatory response, and the neural pathways of pain centers and pain signals in the brain. ${ }^{36,37}$ In addition, a large number of studies have found that there are complex interactions and common genetic risk factors between depression and pain symptoms. ${ }^{12,38}$ Such as, some studies have found that depression can affect changes in multiple brain regions, most commonly in areas such as prefrontal cortex(PFC), anterior cingulate cortex (ACC), hippocampus and amygdala, where gray matter volume loss and alterations in activity similar to those that occur in chronic pain. ${ }^{38,39}$ Neuroimaging 
studies have also revealed the close relationship between brain regions (somatosensory, IC ACC, PFC and thalamus) involved in the integration of pain sensation and emotions and the regions regulated by depression. ${ }^{40}$ These common influencing factors may cause differences in pain susceptibility genes between depression patients and the general population. However, to our knowledge, studies exploring the genetic mechanisms of pain in patients with depression are limited, and the corresponding susceptibility genes remain unclear. Therefore, we conducted GWAS studies on different pain phenotypes in patients with depression, and identified three candidate genes associated with different pain phenotypes. These candidate genes not only have multiple functions affecting psychiatric disorders, nervous system development and neurogenesis, but it is noteworthy that some genes are also involved in physiological processes affecting pain symptoms, such as ADGRF1 identified for neck or shoulder pain, SLC9A9 gene for MCP and TRIOBP for stomach or abdominal pain.

ADGRF1 (adhesion G protein-coupled receptors) is a group of specific target receptors for synaptic amides that are highly expressed in neural stem cells and the brain during development. ${ }^{41}$ Its ligand, N-Docosahexaenoylamine (Synaptamide), is an endocannabinoid metabolite. This signaling pathway can not only by influencing the neurogenic and synaptic genes expression, induction of neurogenesis of hippocampus and cortex neurons and synapses, and the neuronal differentiation of neural stem cells, can also ring through cyclic adenosine monophosphate (cAMP) to transmit anti-inflammatory signals, inhibiting the expression of pro-inflammatory genes and pro-inflammatory cytokines. ${ }^{41,42}$ Because of these biological functions, synaptamide play a role in improving cognitive function and neuronal plasticity in neuropathic pain. ${ }^{43}$ What's more, Park et al. demonstrated the immunomodulatory effects of $A D G R F 1$ in the brain and in the periphery, and jointly promoted the anti-neuroinflammatory effects of synaptamide in systemic inflammatory conditions. These results suggest that ADGRF1 mediated inhibition of innate immune cell activation may be a novel therapeutic strategy for controlling brain and/or peripheral inflammation and related diseases. ${ }^{44}$

SLC9A9(solute carrier family 9, member A9) mainly encodes sodium/proton exchanger 9(NHE9), It is expressed in many brain tissues and modulates luminal $\mathrm{pH}$ of circulating endosomes, which are important organelles for synaptic transmission and plasticity. ${ }^{45}$ And SLC9A9 pathway is associated with long-term potentiation, which underlies cognitive functions that are frequently disrupted in learning and memory, ADHD, and ASD. ${ }^{46}$ Previous studies have found that changes in SLC9A9 gene expression and protein function are associated with a variety of human diseases, such as ADHD, ASD et al. ${ }^{47}$ In addition, a proteinprotein interaction network pathway analysis showed that $S L C 9 A 9$ is also involved in oxidative stress, nociception and other functions. ${ }^{46}$ Since there are multiple overlapping factors between the functions involved in ADGRF1 and SLC9A9 and the influencing factors of pain symptoms, we speculate that two genes are associated with the pain in patients with depression by influencing the factors of pain symptoms. Further studies are still needed to verify our speculation.

TRIOBP gene primarily encodes TRIO and F-actin binding proteins, which are involved in neural tissue development and control actin cytoskeleton organization, cell motility and cell growth. ${ }^{48}$ Among them, several studies have implicated that TRIOBP- 1 is involved in schizophrenia by forming protein aggregates in the brain. ${ }^{49}$ At present, studies of TRIIOBP gene are limited, and further studies are needed to confirm its possible etiological association with pain symptoms.

Among the candidate genes associated with different pain phenotypes identified by PWAS, several candidate genes, such as $P 2 R X 7$ and $S I R P A$, are associated with biological mechanisms that influence pain symptoms or participate in the pain process. $P 2 R X 7$ (purinergic receptor $\mathrm{P} 2 \mathrm{X} 7$ ) is a non-selective cationic channel activated by extracellular ATP. It is mainly expressed in the peripheral and central nervous system and immune system. Activation of $P 2 R X 7$ not only contributes to pro-inflammatory response to injury or bacterial invasion and mediates apoptosis, but also plays a role in chronic inflammation and neuropathic pain. ${ }^{50}$ Such as, an animal study revealed that $P 2 R X 7$ may promote pain modulation through its effect on peripheral tissue damage and altered central nervous system processing in clinical pain states. ${ }^{51}$ Kambur's study reinforces this evidence and suggests that $P 2 R X 7$ gene and genetic variants may be involved in regulating human pain sensitivity. ${ }^{50}$ In addition, previous studies have found that the $P 2 R X 7$ variant is associated with a higher risk of psychiatric disorders such as bipolar disorder and depression, and that the $P 2 R X 7$ receptor is involved in psychosis related pathways, such as synaptic plasticity, neurotransmission, and immune regulation. ${ }^{52}$

It is worth noting that SIRPA gene was overlapping genes in two PWAS studies with different reference weights. SIRPA gene mainly encodes proteins that are members of the signal-regulatory-protein (SIRP) family, which are involved in signal transduction mediated by various growth factor receptors. Previous studies have found that cleavage of signal-regulatory-protein a (SIRPa) is related to enhanced inflammatory signaling, ${ }^{53} \mathrm{CD} 47$ is a demonstrated ligand for this receptor protein, and its signaling pathway, CD47-SIRPa, is involved in the regulation of immune homeostasis and neuronal networks. ${ }^{54}$ What's more, Haiyue Zhang et al revealed the importance of CD47 and SIRPa in the neuroinflammatory process of central nervous system diseases. ${ }^{55}$ Overall, $P 2 R X 7$ has been shown to be directly involved in the regulation of chronic pain, while SIRPA is mainly involved in the biological process of influencing factors of pain symptoms, so it can be speculated that they may influence pain symptoms by regulating and participating in inflammatory responses. Further studies are needed to confirm our speculation. In addition, among the numerous candidate genes identified in the PWAS study, many genes are also associated with psychiatric disorders, nervous system development, and neuronal conduction. For example, PPM1 $F^{56}$ and $G S T M 3^{57}$ et al.

In the calculation results of genetic correlation with different psychiatric disorders, we found that ADHD, and PTSD were significantly positively correlated with different pain phenotypes, reflecting the possible common genetic structure. It is particularly noteworthy that the genetic positive correlation between ADHD and MCP phenotype was consistent with the significant association gene (SLC9A9) identified in the GWAS of MCP. SLC9A9 gene pathway is associated with long-term potentiation, which is the basis of cognitive functions that are frequently disrupted in ADHD. ${ }^{46}$ This further indicates that ADHD and MCP phenotype share the common genetic structure, and also reflects pleiotropy, in which specific genetic alleles may increase the risk of both phenotypes. ${ }^{58}$

In addition, enrichment analysis revealed that significant genes were associated with multiple biological processes and functional pathways. Some of these related biological processes and functional pathways can directly or indirectly affect pain symptoms. For example, sulfur compound metabolism process,

Page $7 / 12$ 
previous studies have found that sulfur compounds (such as hydrogen sulphide) can relieve pain symptoms and inflammation ${ }^{59}$, so significant genes may regulate the pain of patients with depression by participating in sulfur compound metabolism process, What's more, some studies have also found that signal release and chemical synaptic transmission are involved in the biological process of pain symptoms ${ }^{60}$, and the biological relationship between other pathways and pain symptoms needs to be further studied.

Overall, one advantage of our study is the use of a large sample size from the UK Biobank, which can eliminate statistical noise by overcoming potential confounding factors such as selection biases and heterogeneity. In addition, compared with previous pain GWAS studies, our research focused more on multiple pain phenotypes in patients with depression. Based on GWAS, PWAS study and LDSC analysis were further used to explore the potential biological mechanisms related to pain in patients with depression from the perspective of protein expression level and genetics.

Nevertheless, it is worth noting that our research has some limitations. First, all data in our research were derived from the UK Biobank, and the research participants were limited to depression patients of European descent. When applying the research results to different populations, the impact of genetic backgrounds differences on the results should be considered, Secondly, because the phenotyping of pain in the UK Biobank was based on a specific nonstandard pain-related questionnaire, it may result in pain phenotypes being broadly defined and not filtered by other potentially relevant information about the nature, duration or intensity of pain. Finally, genes significantly associated with pain symptoms were identified in our study, and while previous studies have suggested that they play a role in neurological function and disease, there is no direct evidence that they are involved in pain symptoms, and further research is needed to confirm this.

In summary, through GWAS and PWAS analysis, we found several susceptibility genes significantly associated with pain phenotype in patients with depression, What's more, LDSC analysis found that there was a significant genetic correlation between different pain phenotypes and ADHD and PTSD, suggesting that there may be a common genetic structure. These findings contribute to the understanding of the biological mechanism of pain phenotype in patients with depression and provide new clues for the study of its pathogenesis.

\section{Declarations}

Acknowledgments: This study was conducted using the UK Biobank Resource (Application 46478).

Funding: This study was supported by the National Natural Scientific Foundation of China (81922059).

Conflicts of Interest: All authors report no biomedical financial interests or potential conflicts of interest.

Informed Consent Statement: Informed consent was obtained from all subjects involved in the study.

Institutional Review Board Statement: This study has been approved by UK Biobank (Application 46478) and obtained participants' health-related records.

Data Availability Statement: The UK Biobank data are available through the UK Biobank Access Management System https://www.ukbiobank.ac.uk/(accessed on 20 December 2020). We will return the derived data fields following UK Biobank policy, in due course, they will be available through the UK Biobank Access Management System.

Author Contributions: Z.Z. had full access to all the data in the study and takes responsibility for the integrity of the data and the accuracy of the data analysis. Y.W., Y.J. and F.Z. conceptualized and designed the study. All authors contributed in acquisition, analysis, and interpretation of the data. Z.Z. drafted the manuscript. F.Z., L.L. helped with critical revision of the manuscript for important intellectual content. H.Z. performed statistical analysis. Y.W., Y.J. and F.Z. provided administrative, technical, or material support. F.Z. supervised the study. All authors have read and agreed to the published version of the manuscript.

\section{References}

1. Rakel, R. E. Depression. Prim Care 26, 211-224, doi:10.1016/s0095-4543(08)70003-4 (1999).

2. Friedrich, M. J. Depression Is the Leading Cause of Disability Around the World. Jama 317, 1517, doi:10.1001/jama.2017.3826 (2017).

3. Vos, T. et al. Years lived with disability (YLDs) for 1160 sequelae of 289 diseases and injuries 1990-2010: a systematic analysis for the Global Burden of Disease Study 2010. Lancet 380, 2163-2196, doi:10.1016/s0140-6736(12)61729-2 (2012).

4. Lepine, J. P. \& Briley, M. The increasing burden of depression. Neuropsychiatr Dis Treat 7, 3-7, doi:10.2147/NDT.S19617 (2011).

5. Raja, S. N. et al. The revised International Association for the Study of Pain definition of pain: concepts, challenges, and compromises. Pain 161, 19761982, doi:10.1097/j.pain.0000000000001939 (2020).

6. Agüera-Ortiz, L., Failde, I., Mico, J. A., Cervilla, J. \& López-lbor, J. J. Pain as a symptom of depression: prevalence and clinical correlates in patients attending psychiatric clinics. J Affect Disord 130, 106-112, doi:10.1016/j.jad.2010.10.022 (2011).

7. Katona, C. et al. Pain symptoms in depression: definition and clinical significance. Clin Med (Lond) 5, 390-395, doi:10.7861/clinmedicine.5-4-390 (2005).

8. Gerrits, M., van Oppen, P., van Marwijk, H. W. J., Penninx, B. \& van der Horst, H. E. Pain and the onset of depressive and anxiety disorders. Pain 155, 53-59, doi:10.1016/j.pain.2013.09.005 (2014).

9. IsHak, W. W. et al. Pain and Depression: A Systematic Review. Harv Rev Psychiatry 26, 352-363, doi:10.1097/hrp.0000000000000198 (2018).

10. Goesling, J., Clauw, D. J. \& Hassett, A. L. Pain and depression: an integrative review of neurobiological and psychological factors. Curr Psychiatry Rep 15, 421, doi:10.1007/s11920-013-0421-0 (2013). 
11. Bair, M. J., Robinson, R. L., Katon, W. \& Kroenke, K. Depression and pain comorbidity: a literature review. Arch Intern Med 163, 2433-2445, doi:10.1001/archinte.163.20.2433 (2003).

12. Humo, M., Lu, H. \& Yalcin, I. The molecular neurobiology of chronic pain-induced depression. Cell Tissue Res 377, 21-43, doi:10.1007/s00441-019-03003-z (2019).

13. McIntosh, A. M. et al. Genetic and Environmental Risk for Chronic Pain and the Contribution of Risk Variants for Major Depressive Disorder: A FamilyBased Mixed-Model Analysis. PLoS Med 13, e1002090, doi:10.1371/journal.pmed.1002090 (2016).

14. Gasperi, M., Herbert, M., Schur, E., Buchwald, D. \& Afari, N. Genetic and Environmental Influences on Sleep, Pain, and Depression Symptoms in a Community Sample of Twins. Psychosom Med 79, 646-654, doi:10.1097/psy.0000000000000456 (2017).

15. Meng, W. et al. Genetic correlations between pain phenotypes and depression and neuroticism. Eur J Hum Genet 28, 358-366, doi:10.1038/s41431-0190530-2 (2020).

16. Johnston, K. J. A. et al. Genome-wide association study of multisite chronic pain in UK Biobank. PLoS Genet 15, e1008164, doi:10.1371/journal.pgen.1008164 (2019).

17. Sudlow, C. et al. UK biobank: an open access resource for identifying the causes of a wide range of complex diseases of middle and old age. PLoS Med 12, e1001779, doi:10.1371/journal.pmed.1001779 (2015).

18. Davis, K. A. S. et al. Indicators of mental disorders in UK Biobank-A comparison of approaches. Int J Methods Psychiatr Res 28, e1796, doi:10.1002/mpr.1796 (2019).

19. Kroenke, K., Spitzer, R. L. \& Williams, J. B. The PHQ-9: validity of a brief depression severity measure. J Gen Intern Med 16, 606-613, doi:10.1046/j.15251497.2001.016009606.x (2001).

20. Bycroft, C. et al. The UK Biobank resource with deep phenotyping and genomic data. Nature 562, 203-209, doi:10.1038/s41586-018-0579-z (2018).

21. Walter, K. et al. The UK10K project identifies rare variants in health and disease. Nature 526, 82-90, doi:10.1038/nature14962 (2015).

22. Bycroft, C. et al. Genome-wide genetic data on 500,000 UK Biobank participants. bioRxiv, 31, doi:10.1101/166298 (2017).x

23. Purcell, S. et al. PLINK: a tool set for whole-genome association and population-based linkage analyses. Am J Hum Genet 81, 559-575, doi:10.1086/519795 (2007).

24. Bulik-Sullivan, B. K. et al. LD Score regression distinguishes confounding from polygenicity in genome-wide association studies. Nat Genet 47, 291-295, doi:10.1038/ng.3211 (2015).

25. Jansen, I. E. et al. Genome-wide meta-analysis identifies new loci and functional pathways influencing Alzheimer's disease risk. Nat Genet 51, 404-413, doi:10.1038/s41588-018-0311-9 (2019).

26. Otowa, T. et al. Meta-analysis of genome-wide association studies of anxiety disorders. Mol Psychiatry 21, 1391-1399, doi:10.1038/mp.2015.197 (2016).

27. Demontis, D. et al. Discovery of the first genome-wide significant risk loci for attention deficit/hyperactivity disorder. Nat Genet $\mathbf{5 1}, \mathbf{6 3 - 7 5}$, doi:10.1038/s41588-018-0269-7 (2019).

28. Grove, J. et al. Identification of common genetic risk variants for autism spectrum disorder. Nat Genet 51, 431-444, doi:10.1038/s41588-019-0344-8 (2019).

29. Mullins, N. et al. Genome-wide association study of more than 40,000 bipolar disorder cases provides new insights into the underlying biology. Nat Genet 53, 817-829, doi:10.1038/s41588-021-00857-4 (2021).

30. Howard, D. M. et al. Genome-wide meta-analysis of depression identifies 102 independent variants and highlights the importance of the prefrontal brain regions. Nat Neurosci 22, 343-352, doi:10.1038/s41593-018-0326-7 (2019).

31. Nievergelt, C. M. et al. International meta-analysis of PTSD genome-wide association studies identifies sex-and ancestry-specific genetic risk loci. Nat Commun 10, 4558, doi:10.1038/s41467-019-12576-w (2019).

32. Zheng, J. et al. LD Hub: a centralized database and web interface to perform LD score regression that maximizes the potential of summary level GWAS data for SNP heritability and genetic correlation analysis. Bioinformatics 33, 272-279, doi:10.1093/bioinformatics/btw613 (2017).

33. Bennett, D. A. et al. Religious Orders Study and Rush Memory and Aging Project. J Alzheimers Dis 64, S161-s189, doi:10.3233/jad-179939 (2018).

34. Beach, T. G. et al. Arizona Study of Aging and Neurodegenerative Disorders and Brain and Body Donation Program. Neuropathology 35, 354-389, doi:10.1111/neup.12189 (2015).

35. Wingo, A. P. et al. Integrating human brain proteomes with genome-wide association data implicates new proteins in Alzheimer's disease pathogenesis. Nat Genet 53, 143-146, doi:10.1038/s41588-020-00773-z (2021).

36. Pergolizzi, J. et al. The development of chronic pain: physiological CHANGE necessitates a multidisciplinary approach to treatment. Curr Med Res Opin 29, 1127-1135, doi:10.1185/03007995.2013.810615 (2013).

37. Pak, D. J., Yong, R. J., Kaye, A. D. \& Urman, R. D. Chronification of Pain: Mechanisms, Current Understanding, and Clinical Implications. Curr Pain Headache Rep 22, 9, doi:10.1007/s11916-018-0666-8 (2018).

38. Doan, L., Manders, T. \& Wang, J. Neuroplasticity underlying the comorbidity of pain and depression. Neural Plast 2015, 504691, doi:10.1155/2015/504691 (2015).

39. Apkarian, V. A., Hashmi, J. A. \& Baliki, M. N. Pain and the brain: specificity and plasticity of the brain in clinical chronic pain. Pain 152, S49-s64, doi:10.1016/j.pain.2010.11.010 (2011)

40. Fields, H. L. Pain modulation: expectation, opioid analgesia and virtual pain. Prog Brain Res 122, 245-253, doi:10.1016/s0079-6123(08)62143-3 (2000). 
41. Kim, H. Y. \& Spector, A. A. N-Docosahexaenoylethanolamine: A neurotrophic and neuroprotective metabolite of docosahexaenoic acid. Mol Aspects Med 64, 34-44, doi:10.1016/j.mam.2018.03.004 (2018).

42. Raker, V. K., Becker, C. \& Steinbrink, K. The cAMP Pathway as Therapeutic Target in Autoimmune and Inflammatory Diseases. Front Immuno/ 7, 123, doi:10.3389/fimmu.2016.00123 (2016).

43. Tyrtyshnaia, A., Bondar, A., Konovalova, S. \& Manzhulo, I. Synaptamide Improves Cognitive Functions and Neuronal Plasticity in Neuropathic Pain. Int J Mol Sci 22, doi:10.3390/ijms222312779 (2021).

44. Park, T., Chen, H. \& Kim, H. Y. GPR110 (ADGRF1) mediates anti-inflammatory effects of N-docosahexaenoylethanolamine. J Neuroinflammation 16, 225, doi:10.1186/s12974-019-1621-2 (2019).

45. Zhang-James, Y., DasBanerjee, T., Sagvolden, T., Middleton, F. A. \& Faraone, S. V. SLC9A9 mutations, gene expression, and protein-protein interactions in rat models of attention-deficit/hyperactivity disorder. Am J Med Genet B Neuropsychiatr Genet 156b, 835-843, doi:10.1002/ajmg.b.31229 (2011).

46. Zhang-James, Y. et al. Effect of disease-associated SLC9A9 mutations on protein-protein interaction networks: implications for molecular mechanisms for ADHD and autism. Atten Defic Hyperact Disord 11, 91-105, doi:10.1007/s12402-018-0281-x (2019).

47. Patak, J., Faraone, S. V. \& Zhang-James, Y. Sodium hydrogen exchanger 9 NHE9 (SLC9A9) and its emerging roles in neuropsychiatric comorbidity. Am J Med Genet B Neuropsychiatr Genet 183, 289-305, doi:10.1002/ajmg.b.32787 (2020).

48. Zaharija, B., Samardžija, B. \& Bradshaw, N. J. The TRIOBP Isoforms and Their Distinct Roles in Actin Stabilization, Deafness, Mental Illness, and Cancer. Molecules 25, doi:10.3390/molecules25214967 (2020).

49. Bradshaw, N. J. et al. Aggregation of the protein TRIOBP-1 and its potential relevance to schizophrenia. PLoS One 9, e111196, doi:10.1371/journal.pone.0111196 (2014).

50. Kambur, O. et al. Genetic variation in P2RX7 and pain tolerance. Pain 159, 1064-1073, doi:10.1097/j.pain.0000000000001188 (2018).

51. Chessell, I. P. et al. Disruption of the P2X7 purinoceptor gene abolishes chronic inflammatory and neuropathic pain. Pain 114, 386-396, doi:10.1016/j.pain.2005.01.002 (2005).

52. Boks, M. P. et al. Cannabinoids and psychotic symptoms: A potential role for a genetic variant in the P2X purinoceptor 7 (P2RX7) gene. Brain Behav Immun 88, 573-581, doi:10.1016/j.bbi.2020.04.051 (2020).

53. Londino, J. D., Gulick, D., Isenberg, J. S. \& Mallampalli, R. K. Cleavage of Signal Regulatory Protein a (SIRPa) Enhances Inflammatory Signaling. J Biol Chem 290, 31113-31125, doi:10.1074/jbc.M115.682914 (2015).

54. Matozaki, T., Murata, Y., Okazawa, H. \& Ohnishi, H. Functions and molecular mechanisms of the CD47-SIRPalpha signalling pathway. Trends Cell Bio/ 19, 72-80, doi:10.1016/j.tcb.2008.12.001 (2009).

55. Zhang, H., Li, F., Yang, Y., Chen, J. \& Hu, X. SIRP/CD47 signaling in neurological disorders. Brain Res 1623, 74-80, doi:10.1016/j.brainres.2015.03.012 (2015).

56. Liu, J. et al. PPM1F in hippocampal dentate gyrus regulates the depression-related behaviors by modulating neuronal excitability. Exp Neuro/340, 113657, doi:10.1016/j.expneurol.2021.113657 (2021).

57. Maes, O. C., Schipper, H. M., Chong, G., Chertkow, H. M. \& Wang, E. A GSTM3 polymorphism associated with an etiopathogenetic mechanism in Alzheimer disease. Neurobiol Aging 31, 34-45, doi:10.1016/j.neurobiolaging.2008.03.007 (2010).

58. Pingault, J. B. et al. Using genetic data to strengthen causal inference in observational research. Nat Rev Genet 19, 566-580, doi:10.1038/s41576-0180020-3 (2018).

59. Distrutti, E. Hydrogen sulphide and pain. Inflamm Allergy Drug Targets 10, 123-132, doi:10.2174/187152811794776240 (2011).

60. Woolf, C. J. \& Salter, M. W. Neuronal plasticity: increasing the gain in pain. Science 288, 1765-1769, doi:10.1126/science.288.5472.1765 (2000).

\section{Figures}




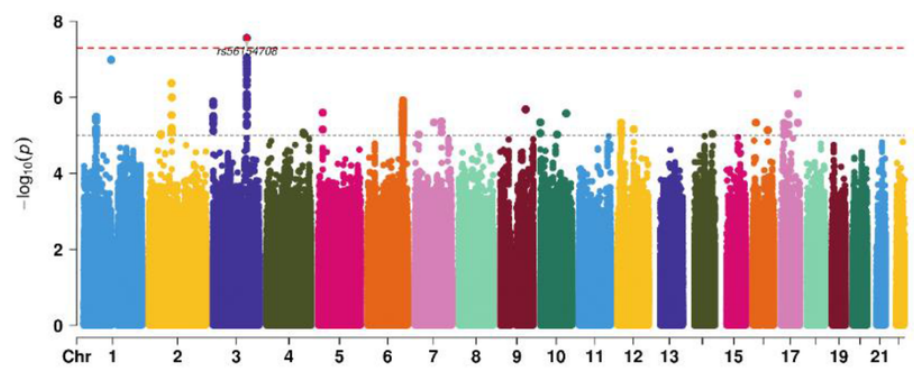

(a)

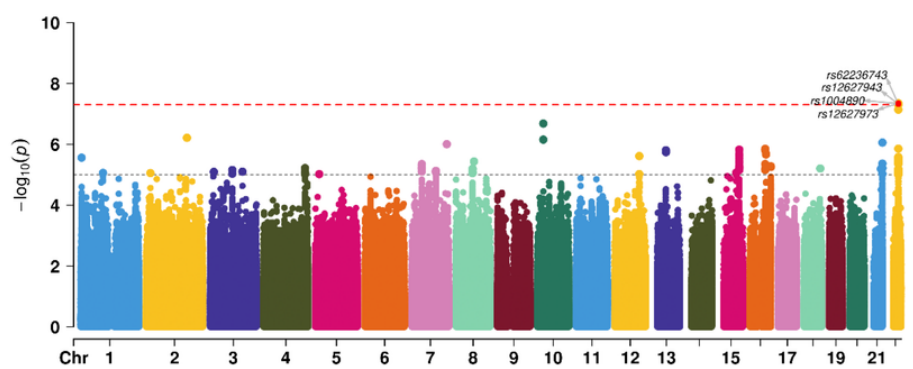

(b)

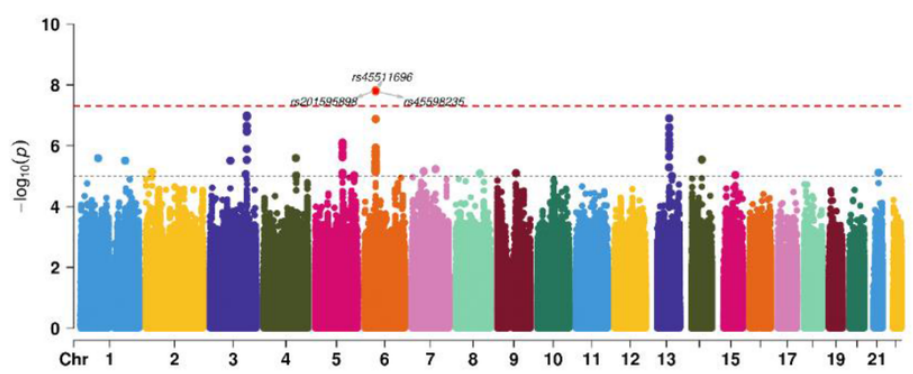

(c)

\section{Figure 1}

Manhattan plot of different pain phenotypes in patients with depression. (a) Multisite chronic pain, a SNP allele was found to be significantly associated with MCP (b) Stomach or abdominal pain phenotype, four independent SNP alleles were significantly associated with stomach pain or abdominal pain phenotype. (c) Neck or shoulder pain phenotype, three independent SNP alleles were significantly associated with Neck or shoulder pain phenotype. Biobank Genomic coordinates are displayed along the X-axis, with the negative logarithm of the association P value for each SNP displayed on the Y-axis, meaning that each dot on the Manhattan plot signifies a SNP. The red line indicates the P-value threshold for genome-wide significance $\left(P_{G W A S}<5 \times 10^{-8}\right)$ while the blue line indicates $\mathrm{P}$-value threshold for suggestive significance $\left(\mathrm{P}_{\mathrm{GWAS}}<1 \times 10^{-5}\right)$.

\section{Figure 2}

PWAS results of Banner reference weights for different pain phenotypes in patients with depression. (a) Multisite chronic pain, 17 candidate genes were found to be significantly associated with MCP. (b) Stomach or abdominal pain phenotype, 16 candidate genes were significantly associated with stomach pain or abdominal pain phenotype. (c) Neck or shoulder pain phenotype, 8 candidate genes were significantly associated with Neck or shoulder pain phenotype. The results of the PWAS study are shown through the Manhattan plot. These candidate genes may contribute to the biological mechanisms of pain in depressed patients through their cis-regulated brain protein abundance. The red line indicates the $\mathrm{P}$-value threshold for Proteome-wide significance $(\mathrm{P}<0.05)$.

\section{Figure 3}

PWAS results of Rosmap reference weights for different pain phenotypes in patients with depression. (a) Multisite chronic pain, 22 candidate genes were found to be significantly associated with MCP. (b) Stomach or abdominal pain phenotype, 15 candidate genes were significantly associated with stomach pain or abdominal pain phenotype. (c) Neck or shoulder pain phenotype, 14 candidate genes were significantly associated with Neck or shoulder pain phenotype. The results of the PWAS study are shown through the Manhattan plot. These candidate genes may contribute to the biological mechanisms of 
pain in depressed patients through their cis-regulated brain protein abundance. The red line indicates the P-value threshold for Proteome-wide significance ( $\mathrm{P}$ $<0.05)$.

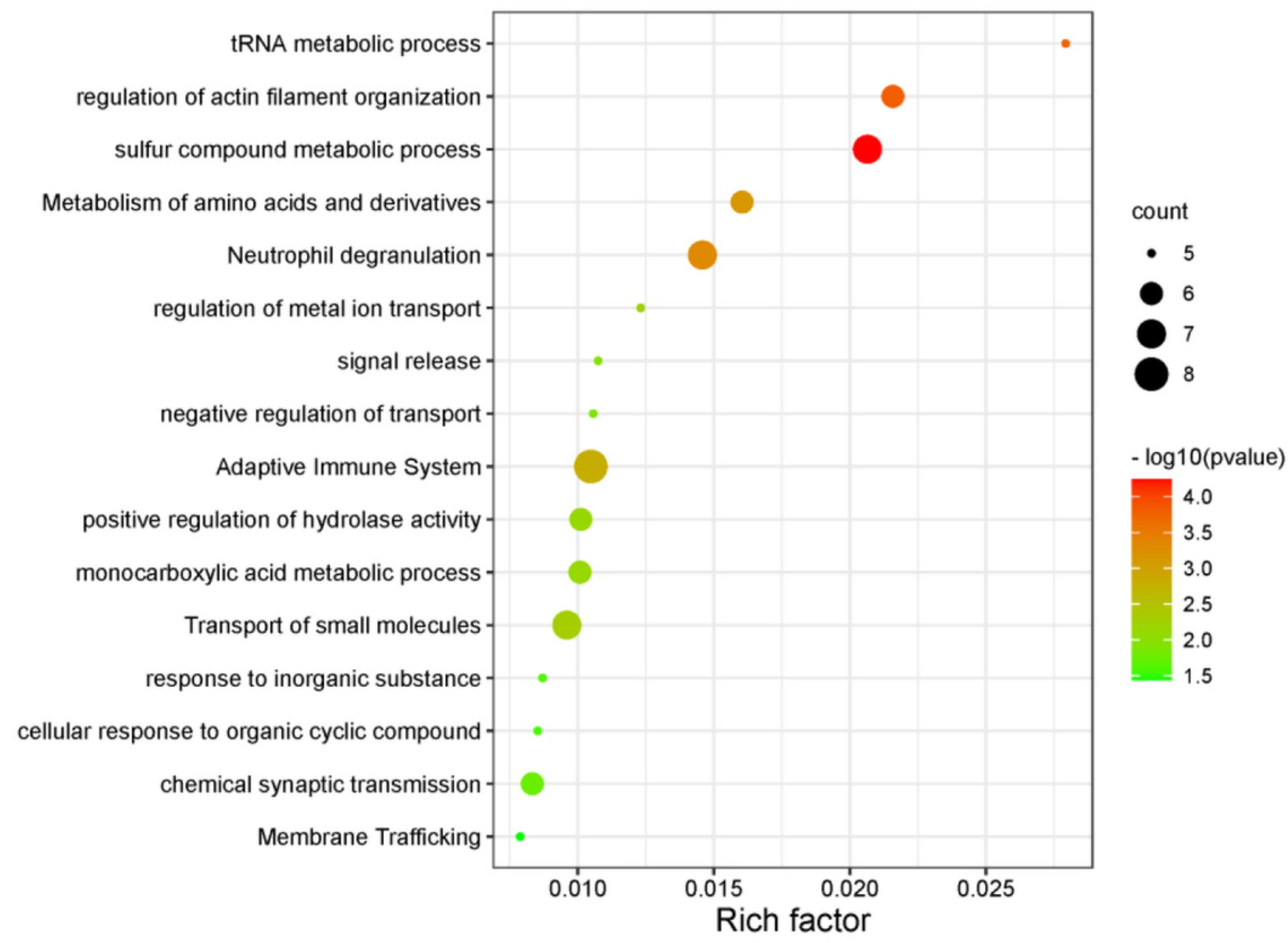

Figure 4

Bubble plot of enrichment analysis. This bubble plot showed biological processes or functional pathways of the 16 clusters in the enrichment analysis results.

\section{Supplementary Files}

This is a list of supplementary files associated with this preprint. Click to download.

- TheSupportingInformation.docx 